\title{
Simultaneous screening of antibiotic residues in honey by biochip multi-array technology
}

\author{
AKSEM AKSOY \\ Department of Food Engineering, Faculty of Engineering Architecture, Kafkas University, Kars, Turkey
}

\section{Aksoy A. \\ Simultaneous screening of antibiotic residues in honey by biochip multi-array technology}

Summary

The existence of antibiotic residues in food causes serious health problems in humans. This study aims to determine antibiotic residues in honey intended for consumption. Evidence Investigator TM Biochip Array technology enables simultaneous quantitative detection of multiple analytes on a single sample. The Anti Microbial Array IV test kit is used in simultaneously detecting the quantity of 12 different groups of antimicrobials in honey samples. A competitive chemiluminescent immunoassay was employed for the detection of antimicrobials. The light signal produced from each test region on the biochip was detected using digital imaging technology. In this study, a total of $\mathbf{4 5}$ honey samples including 5 brands of honey and 40 local honey were analyzed. The honey samples were simultaneously screened for 12 different types of antibiotic residues by using the Evidence Investigator ${ }^{\mathrm{TM}}$ Anti Microbial Array IV test kit. In the honey samples examined, residues of erythromycin, streptomycin, amikacin, lincosamides, tylosin B and neomycin were detected. $91 \%$ of samples involved erythromycin residues whereas $15 \%$ of the local samples included streptomycin residues above the recommended concentration.

Keywords: biochip, multi-array, antibiotic residues, screening, honey

The main components of honey are carbonhydrates whereas honey also involves lower quantities of water and many smaller components (3). Honey is a natural sweetener having a complex composition which may vary by plant source, geographic origin, climate, as well as processing and storage conditions. Honey which largely consists of carbonhydrates and water also includes nitrogen compounds, organic acids, minerals, vitamins, Maillard reaction products, volatile compounds and bioactive substances in low quantities (7).

Honey, produced by honeybees as a valuable food product, may lose quality and be harmful to human health if it involves xenobiotics. Antibiotics are commonly used by beekeepers for control of diseases. Common use of antibiotics causes bacteria to become resistant to many drugs and the expansion of antibioticresistant bacteria strains (2). Globally, antibiotic use is one of the most important factors that cause antibiotic resistance. Antibiotics are prescription drugs frequently used in human medicine. They are also commonly used to prevent, control and treat animal diseases and promote growth in food-producing animals. It is not easy to directly compare the amount of drugs used in food animals with the amount used in humans; nevertheless, it is known that there is evidence of higher use of anti- biotics in food production (10). Use of antibiotics for the control of bacterial diseases in beekeeping causes antibiotic residues in honey. The primary reasons for the existence of antibiotic residues in honey are the environment and uninformed beekeeping practices. Oxytetracycline is commonly used in the treatment of European foulbrood and American foulbrood whereas erythromycin, lincomycin, monensin, streptomycin and enrofloxacin are also used $(1,17)$.

Honey or any other food should not involve antibiotics due to risks to human health. They cause allergy risk and formation of resistant strains in human pathogens even in very low doses. Due to these concerns, many countries have banned or restricted the use of antimicrobial compounds in food-producing animals, and Maximum Residue Limits (MRL) for antimicrobial residues in food have been set (11). The European Union does not allow the use of any veterinary medical products involving antibiotics in beekeeping. There is no MRL for antibiotics in honey $(5,11,14,19)$. Some countries such as Switzerland, the UK and Belgium have set action limits for antibiotics in honey which vary between 0.01 and $0.05 \mathrm{mg} / \mathrm{kg}$ for each antibiotic group. Antibiotic residues have been reported throughout the world in various honey samples. Therefore, 
there is a need for adequate tracking and reliable implementation methods (14).

The use of veterinary drugs in animals for treatment and especially prevention purposes cause antibiotic residues to exist in animal food substances. It is necessary for the safety of consumers to control veterinary drug residues in food. Screening methods are the first step in controlling antibiotic residues in food (12). There are various techniques for screening residues in foods of animal origin, the main ones being immunological methods: ELISA test kits, radioimmunoassay, multiarray biosensors $(4,11,14,16,18,20,22,34)$ and chromatographic methods: high performance thinlayer chromatography (HPTLC), or high performance liquid chromatography (HPLC) $(26,28,35)$. Biochip array biosensors have been commonly used due to reasons such as ease of use, rapid results, simultaneous multi-residue analysis, full automation and high efficiency (33).

Analytic systems and the latest technological developments have provided new platforms with more automation and multiplexing ability than conventional biological binding tests. Multiplexed bioanalytical techniques provide new opportunities for food industries and control agents to more effectively monitor and improve food and environmental contaminants (27). Microarray technology is a strong analytical tool for simultaneously detecting multiple analytes in a single sample and is a developing field in analytical chemistry. A microarray consists of a reactive dot matrix on a supporting material. Multi-analyte immunoassays on microarrays and multiplex DNA microarrays have been identified for the quantitative analysis of antibiotics, small organic molecules such as small molecule toxins, proteins, microorganisms, viruses and eukaryotic cells (30).

The technical guidelines published by the European Union Reference Laboratories (6) recommend concentrations for the residue analysis of certain antibiotics in honey. However, these concentrations have no actual legal basis $(11,19)$. The recommended concentrations are $40 \mu \mathrm{g} / \mathrm{kg}$ for streptomycin, $20 \mu \mathrm{g} / \mathrm{kg}$ for tetracyclines, $50 \mu \mathrm{g} / \mathrm{kg}$ for sulphonamides, and $20 \mu \mathrm{g} / \mathrm{kg}$ for erythromycin and tylosin (6).

The aim of this study was to detect and assess the existence of probable residues of 12 different types of antibiotics simultaneously on honey samples presented for consumption by means of biochip multi-array technology.

\section{Material and methods}

Samples. In this study, a total of 45 honey samples including 5 brands of honey and 40 local honey (honey produced in the region by beekeepers) were analyzed. The honey samples of different brands were supplied from supermarkets in Kars province whereas local honey was randomly collected from honey sales points in the city. The samples were delivered into sterile sample bags (at least
$100 \mathrm{~g}$ ) and maintained under room temperature in a closed environment to protect them from heat, light and humidity until the day of analysis.

Anti Microbial Array IV kit (AM IV). Anti Microbial Array IV kit is used in detecting multiple aminoglycoside and macrolide groups of antibiotics which are specifically found in honey. It simultaneously detects on a single honey sample 12 different types of antibiotics: namely spiramycin (SPR), apramycin (APA), bacitracin (BCT), neomycin (NEO), tobramycin (TOB), tylosin B (TYB), spectinomycin (SPT), amikacin (AMK), lincosamides (LIN), erythromycin (ERY), streptomycin (STR) and virginiamycin (VIR).

Sample preparation. The samples were prepared in accordance with the instructions of the producing company (25). $1 \mathrm{~g}$ of honey was weighed and $9 \mathrm{ml} \mathrm{K}_{2} \mathrm{HPO}_{4}(\mathrm{pH} 8.0)$ was added. $10 \mathrm{ml}$ diluted washing solution was added and vortexed for $1 \mathrm{~min}$ or until the sample was completely dissolved to make it suitable to be applied to a biochip. Dilution factor $=20$.

Biochip analysis. The Evidence Investigator ${ }^{\mathrm{TM}}$ Biochip Array technology is used to perform simultaneous quantitative detection of multiple analytes from a single sample. The core technology is the Randox Biochip, a solid-state device with an array of discrete test regions containing immobilised antibodies specific to different marcolides and aminoglycosides. A competitive chemiluminescent immunoassay is employed for the anti microbial array. Increased levels of marcolides and aminoglycosides in a specimen will lead to decreased binding of marcolides and aminoglycosides labelled with horseradish peroxidase (HRP) and thus a decrease in chemiluminescence being emitted. Light is formed by chemiluminescence. Luminol and Peroxide (Signal reagent) creates a light reaction. A nine-point calibration was performed using the Randox Evidence Investigator ${ }^{\mathrm{TM}}$ Anti Microbial Array IV calibrators, which cover the calibration range of all assays. A maximum of 6 biochip carriers may be assayed simultaneously, and it is recommended that a new calibration curve be constructed for each assay series.

All analyzes were performed according to the manufacturer's instructions (25). The solutions required for the test were prepared in accordance with the suggestions of the producing company and all materials were brought to room temperature. The samples were analyzed by an Evidence Investigator Anti Microbial Array IV kit (EV 3878, Randox Laboratories Ltd., Crumlin, County Antrim, UK). $100 \mu 1$ test buffer was pipetted into the wells. Next, a $100 \mu$ l calibrator or sample was pipetted into the wells. To mix the reactants, all sides of the plate were tapped and the holding plate was fixed onto the bottom plate of the thermo shaker and incubated for $30 \mathrm{~min}$ at $25^{\circ} \mathrm{C}$ and $370 \mathrm{rpm} .100 \mu \mathrm{l}$ conjugate per well was pipetted. It was incubated in the thermo shaker for $60 \mathrm{~min}$ at $25^{\circ} \mathrm{C}$ and $370 \mathrm{rpm}$. The reactants were removed by sharply moving the process plate. Two rapid washing processes were immediately performed with about $350 \mu \mathrm{l}$ diluted washing solution per well. The washing cycle was performed 4 more times. For each cycle, all sides of the process plate were tapped for about 10 to $15 \mathrm{sec}$, then the biochips were kept in the washing buffer for $2 \mathrm{~min}$. After the final wash, the wells were filled with washing buffer and kept there until direct imaging. The imaging process was 
conducted within $30 \mathrm{~min}$. The results were automatically assessed in the Randox Evidence Investigator software. Evidence Investigator (Randox Laboratories Ltd., Crumlin, County Antrim, UK) identifies images by using Relative Light Units (RLU) which conducts the reading process via Charge Coupled Device (CCD) camera at a temperature of $-40^{\circ} \mathrm{C}$. The concentration of the analytes in the sample was found by the Evidence Investigator ${ }^{\mathrm{TM}}$ Anti Microbial Array IV (EV 3878, Randox Laboratories Limited, Crumlin, County Antrim, UK). Anti Microbial Array IV kit has been validated by the manufacturer as a result of validation studies with reference samples.

\section{Results and discussion}

None of the 45 honey samples analyzed in the study were detected with spiramycin, apramycin, bacitracin, tobramycin, spectinomycin or virginiamycin antibiotic residues. In $41(91.1 \%)$ samples, erythromycin was found. $11(24.4 \%)$ samples had neomycin, $10(22.2 \%)$ had streptomycin, $8(17.8 \%)$ had amikacin, $6(13.3 \%)$ had lincosamides, and $2(4.4 \%)$ had tylosin B residues. In the branded honey samples, $4(80 \%)$ samples were detected with erythromycin residue whereas $1(20 \%)$ had streptomycin and $1(20 \%)$ had neomycin residues. Among the local honey samples, $37(92.5 \%)$ had erythromycin, $9(22.5 \%)$ had streptomycin, $6(15 \%)$ had lincosamides, $8(20 \%)$ had amikacin, $2(5 \%)$ had tylosin B and $10(25 \%)$ had neomycin residues. The results obtained are shown on Table 1 in detail.

Antibiotics are primarily used against microbial infections and also constitute a significant cause of death worldwide. Many antibiotics used as therapeutic and growth inducing agents in animals may cause antibiotic residues in foods of animal origin thereby harming human health. Therefore, it is vital to screen foods of animal origin for antibiotic residues (4).

Tab. 1. Antimicrobial substances detected in honey samples $(n=45)$ by biochip multi-array analysis (ppb)

\begin{tabular}{|c|c|c|c|c|}
\hline \multirow[t]{2}{*}{ Antimicrobials } & \multirow{2}{*}{ LOD* (ppb) } & \multirow{2}{*}{$\begin{array}{c}\text { Number of } \\
\text { positive samples } \\
n(\%)\end{array}$} & \multicolumn{2}{|c|}{$\begin{array}{l}\text { Antibiotic concentration } \\
\text { in positive samples (ppb) }\end{array}$} \\
\hline & & & Mean \pm SD & $\min -\max$ \\
\hline Spiramycin & 2.0 & not detected & - & - \\
\hline Apramycin & 2.0 & not detected & - & - \\
\hline Bacitracin & 1.2 & not detected & - & - \\
\hline Neomycin & 1.0 & $11(24.4)$ & $0.81 \pm 2$ & $<1.0^{*}-8.4$ \\
\hline Tobramycin & 4.0 & not detected & - & - \\
\hline Tylosin B & 1.0 & $2(4.4)$ & $1.45 \pm 8.3$ & $<1.0^{*}-55.2$ \\
\hline Spectinomycin & 2.6 & not detected & - & - \\
\hline Amikacin & 6.0 & $8(17.8)$ & $4.11 \pm 9.4$ & $<1.0^{*}-33.7$ \\
\hline Lincosamides & 8.0 & $6(13.3)$ & $1.23 \pm 3.2$ & $<1.0^{*}-10.7$ \\
\hline Erythromycin & 2.5 & 41 (91.1) & $6.71 \pm 5.6$ & $<1.0^{*}-38.1$ \\
\hline Streptomycin & 4.0 & $10(22.2)$ & $59.9 \pm 213.8$ & $<1.0^{*}-1000$ \\
\hline Virginiamycin & 2.0 & not detected & - & - \\
\hline
\end{tabular}

Explanations: *LOD - lowest limit of detection; $<1.0 *$ - below minimum detection limit (LOD) for detected and quantified antimicrobial substance
In a study on honey, Sunay (31) reported that $75 \%$ of the honey examined was free from sulfadimidine, tetracycline and streptomycin residues whereas $90 \%$ involved streptomycin residues at rates lower than $10 \mathrm{ppb}$. In a study examining streptomycin residues in a total of 56 samples obtained from different regions in Turkey in 2011 and 2012, Seğmenoğlu (29) reported that $92.8 \%$ of the honey examined had no streptomycin. In another study by Zai et al. (35) tetracycline, streptomycin, gentamicin and penicillin antibiotic residues and their respective quantities were determined by using the TLC method and the positive samples were optimized by HPLC. About $12.5 \%$ of the branded samples and $19.9 \%$ of the unbranded samples were found to be positive. They reported that in the samples tested by the TLC method, no gentamicin was found whereas in the unbranded samples, the tetracycline residue was at the maximum level. In the HPLC analysis, the total streptomycin residue was determined as $16.31 \mu \mathrm{g} / \mathrm{g}$ in five positive unbranded samples and found at minimum $(3.6 \mu \mathrm{g} / \mathrm{ml})$. The researchers emphasized that unbranded honey showed higher antibiotics residue contamination than branded honey. This study found $22.2 \%$ of the branded and local honey samples positive in terms of streptomycin residues. In $15 \%$ of the local honey samples, the streptomycin residue level exceeded the recommended concentration. This is quite high compared to the findings of the abovementioned study. This result points to the fact that the beekeepers in the region have been using antibiotics involving streptomycin. In a study conducted by Rao et al. (26) the entirety of the branded and raw honey samples examined showed oxytetracycline residues and the oxytetracycline concentration was higher in the raw honey samples. Saleh et al. (28) reported that they detected oxytetracycline residues in $31.2 \%$ of the honey samples examined and tetracycline residues in $12.5 \%$ of the samples, and in all samples, MRL $_{\mathrm{s}}$ were higher than the international standards.

In $41(91.1 \%)$ of the honey samples examined in this study (branded and local honey), erythromycin residues were found. A study which analyzed erythromycin residues in 50 honey samples collected from the South Marmara region of Turkey, reported detection and quantification limits as 6 and $20 \mathrm{ngg}^{-1}$ respectively and the existence of erythromycin residues in varying concentrations from 50 to $1776 \mathrm{ng} \mathrm{g}^{-1}$ in four (8\%) honey samples (13). The results we have obtained are considerably higher than the results of the above-mentioned study. This shows that in the region where the samples were collected, beekeepers have been commonly using antibiotics 
involving erythromycin in the treatment of diseases. In $6(15 \%)$ of the local honey, lincosamides residues were found. This shows that the lincosamides group of antibiotics are also used in beekeeping in the region. In a study conducted by Tamba-Berehoiu et al. (32) examining antibiotic residues in honey presented for consumption, except for the linden honey samples obtained from Bacau, in all honey samples analyzed, streptomycin residues ( $40 \mathrm{ppb}$ ) were found above the recommended concentrations. Tetracycline residues were found in all samples, and only the acacia honey from Moinești and linden honey from Onești had them above the recommended concentration whereas the level of erythromycin residues did not exceed the recommended concentration in any of the samples. In this study, $41(91.1 \%)$ of the samples showed erythromycin residues and only in one local sample was the rate above the recommended concentration. In this study, 2 of the local honey samples had tylosin B residues. In a study in which Mahmoudi et al. (18) determined antibiotic residues in honey in Iran using the ELISA method, it was reported that the samples obtained in the summer and autumn were detected with tylosin residues whereas the samples obtained in the spring had no tylosin residues.

The majority of the researchers have examined the existence of antibiotics residues by biochip array technology (16, 20, 23, 24, 34). O'Mahony et al. (20) screened the residues of nitrofuran antibiotics which should not exist in honey by a chemiluminescencebased biochip array sensing technique. They reported that the biochip array method allowed for the detection of all four metabolites below the reference point and that the biosensor method had the potential to be a suitable screening technique in the field of food safety technologies. Similarly, Li et al. (16) reported that the microarray method had the potential to become a relevant screening technique in the food safety field. Another study that used the antimicrobial Array II kit simultaneously screened six antibiotic families in various types of honey. It was reported that the method was swift and the sample preparation procedure was simple. The researchers indicated that according to the results obtained, the suggested method was suitable for the screening analysis of six antibiotic families (11). In another study, it was reported that the detection limits for streptomycin, dihydrostreptomycin and kanamycin were as low as $0.993,0.913$ and $1.23 \mu \mathrm{gkg}^{-1}$ respectively and it was emphasized that the method used was suitable for the extraction of aminoglycoside antibiotics in honey samples (15).

In a study in which Oruç et al. (21) determined the existence of 6 different groups of antibacterial drugs in oral fluids of pigs collected in experimental conditions by biochip array-based immunoassay method, it was reported that norfloxacin, ceftiofur, florfenicol, streptomycin, tylosin and tetracycline were detected in clean and dirty oral fluids of pigs. They reported that tetracycline recovery was quite low in dirty oral fluid; thus, except for tetracycline screening in dirty oral fluid, they could readily detect target antibacterial drugs of six different groups in oral fluid of pigs through this method. The results of another study in which six different groups of antibacterial drugs were detected in milk, meat, urine and forage under experimental conditions reported that the biochip array-based immunoassay method may be used in the routine analysis of antibacterial drug residues in these samples (22).

The emergence of antibiotic-resistant species throughout the world has caused an increase in studies dealing with the research and detection of antibiotic residues. It is important to periodically screen antibiotics in foods of animal origin for the protection of human health and food safety. For this aim, the use of rapid screening tests is advantageous. In this regard, it is very important that the Antimicrobial Array IV kit used in this study has the capacity to simultaneously screen 12 different types of antibiotics.

Food animals are considered as the main reservoirs of antibiotic-resistant bacteria due to antibiotic use in food production industry (9). A significant finding of this study is the detection of multiple antibiotics in a single sample. The determination of both aminoglycoside and macrolide antibiotic residues in the same samples brings about the issue of antibiotic resistance. Consumption of honey under these circumstances may cause the formation of resistant species. As a result of a study investigating antibiotic residue levels in human breast milk, Dinleyici et al. (8) reported that $85.7 \%$ of the human breast milk samples of mothers who did not take any antibiotics at birth or in the first seven days after birth had beta-lactam and $23.5 \%$ had quinolone residues. They pointed out that efficient policies are needed for appropriate antibiotic use during pregnancy and lactation as well as in food safety.

Consequently, the antimicrobial IV Biochip test designed to determine multiple aminoglycoside and macrolide group of antibiotics that may be found in honey, can simultaneously detect these compounds in a single honey sample. It is very important for human health to routinely screen honey for antibiotic residues. Therefore, this study shows that the ability of the multiplexing screening technology used in this study to detect 12 different types of antibiotic residues in a short time will be very advantageous for food safety and useful in different fields of the food industry. According to the screening analyses conducted, erythromycin, streptomycin, neomycin, tylosin B, amikacin and lincomycin residues were found in the honey samples in various concentrations. This outcome points to the fact that there is uncontrolled antibiotic use in beekeeping. The detection of various antibiotics in a single sample brings to mind antibiotic resistance problems that may come up upon consumption of animal food products. Therefore, the drugs used in beekeeping must be kept 
under strict control. Beekeepers should be informed about the adverse effects of uninformed antibiotic use on public health, and training programs in this regard should be prioritized. The actualization of informed and principled beekeeping practices will secure public health.

\section{References}

1. Al-Waili N., Salom K., Al-Ghamdi A., Ansari M. J.: Antibiotic, pesticide, and microbial contaminants of honey: human health hazards. Scient. World J. 2012, Article ID 930849, 1-9.

2. Barganska Z., Slebioda M., Namiesnik J.: Determination of antibiotic residues in honey. Trends Anal. Chem. 2011, 30, 1035-1041.

3. Bogdanov S.: Honey composition. Bee Prod. Sci. 2009, 1-10.

4. Chen T., Cheng G., Ahmed S., Wang Y., Wang X., Hao H., Yuan Z.: New methodologies in screening of antibiotic residues in animalderived foods: Biosensors. Talanta. 2017, 175, 435-442.

5. Commission E.: Commission Regulation (EU) No 37/2010 of 22 December 2009. OJEC 2010, L15, 1-72.

6. CRL.: Guidance paper of $7^{\text {th }}$ December 2007. CRLs view on state of the art analytical methods for national residue control plans. Community Reference Laboratories (CRLs) for residues according to Council Directive 96/23/EC. $1-8$

7. De-Melo A. A. M., de Almeida-Muradian L. B., Sancho M. T., Pascual-Maté A.: Composition and properties of Apis mellifera honey: A review. J. Apic. Res. 2018, 57, 5-37.

8. Dinleyici M., Yıldırım G. K., Aydemir O., Kaya T. B., Bildirici Y., Carman $K$. B.: Human milk antibiotic residue levels and their relationship with delivery mode, maternal antibiotic use and maternal dietary habits. Eur. Rev. Med. Pharmacol. Sci. 2018, 22, 6560-6566.

9. Founou L. L., Founou R. C., Essack S. Y.: Antibiotic resistance in the food chain: a developing country-perspective. Front. Microbiol. 2016, 7, 1881.

10. Frieden T.: Antibiotic resistance threats in the United States. U.S. Departmen of health and human services. Centers for disease control and prevention. 2013 1-12.

11. Gaudin V., Hedou C., Soumet C., Verdon E.: Evaluation and validation of biochip multi-array technology for the screening of six families of antibiotics in honey according to the European guideline for the validation of screening methods for residues of veterinary medicines. Food Addit. Contamin. Part A 2014, 31, 1699-1711.

12. Gaudin $V$ : Advances in biosensor development for the screening of antibiotic residues in food products of animal origin-A comprehensive review. Biosens. Bioelectron. 2017, 90, 363-377.

13. Günes N., Clbık R., Günes M. E., Aydın L.: Erythromycin residue in honey from the Southern Marmara region of Turkey. Food Addit. Contamin. Part A 2008, 25, 1313-1317.

14.Jakšić S. M., Ratajac R. D., Prica N. B., Apić J. B., Ljubojević D. B., Žekic Stošić M. Z., Živkov Baloš M. M.: Methods of determination of antibiotic residues in honey. J. Anal. Chem. 2018, 73, 317-324.

15. Li D., Li T., Wang L., Ji S.: A polyvinyl alcohol-coated core-shell magnetic nanoparticle for theextraction of aminoglycoside antibiotics residues from honey samples. J. Chromatogr. A 2018, 1581-1582, 1-7.
16. Li Z. H., Li Z. M., Xu D. K.: Simultaneous detection of four nitrofuran metabolites in honey by using a visualized microarray screen assay. Food Chem. 2017, 221, 1813-1821.

17. Mahmoudi R., Ghojoghi A., Ghajarbeygi P.: Honey safety hazards and public health. JCHR. 2016, 6, 249-267.

18. Mahmoudi R., Norian R., Pajohi-Alamoti M. R.: Antibiotic residues in Iranian honey by ELISA. Int. J. Food Proper. 2014, 17, 2367-2373.

19. Moretti S., Saluti G., Galarini R.: Residue determination in honey, honey analysis. Prof. Vagner Arnaut De Toledo (Ed.), InTech 2017, p. 326.

20. O'Mahony J., Moloney M., McConnell R. I., Benchikh E. O., Lowry P., Furey A., Danaher M.: Simultaneous detection of four nitrofuran metabolites in honey using a multiplexing biochip screening assay. Biosens. Bioelectron. 2011, 26, 4076-4081

21. Oruç H. H., Rumbeiha W. K., Ensley S., Olsen C., Schrunk D. E. Simultaneous detection of six different groups of antimicrobial drugs in porcine oral fluids using a biochip array-based 1mmunoassay. Kafkas Univ. Vet. Fak. Derg. 2013, 19, 407-412.

22. Oruç H. H., Rumbeiha W. K., Ensley S., Schrunk D. E.: Simultaneous detection of six different groups of antimicrobial drugs in milk, meat, urine, and feed matrices. Uludağ Univ. J. Fac. Vet. Med. 2012, 31, 29-33.

23. Plotan M., Devlin R., Porter J., Benchikh M. E. O., Rodríguez M. L., McConnell R. I., FitzGerald S. P.: The use of biochip array technology for rapid multimycotoxin screening. J. AOAC Int. 2016, 99, 878-889.

24. Popa I. D., Schiriac E. C., Cuciureanu R.: Multi-analytic detection of antibiotic residues in honey using a multiplexing biochip assay. Rev. Med. Chir. Soc. Med. Nat. Iasi. 2012, 116, 324-329.

25. Randox Manual: Antimicrobial Array IV (AM IV), EV3878 Manual, 2018.

26. Rao C. R. M., Kumar L. C. A., Sekharan C. B.: Quantitative analysis of oxytetracycline residues in honey by high performance liquid chromatography. Int. Res. J. Biol. Sci. 2015, 4, 59-65.

27. Raz S. R., Haasnoot W.: Multiplex bioanalytical methods for food and environmental monitoring. Trends Anal. Chem. 2011, 30, 1526-1537.

28. Saleh S. M. K., Mussaed A. M., Al-Hariri F. M.: Determination of tetracycline and oxytetracycline residues in honey by high performance liquid chromatography. JAST B 2016, 6, 135-139.

29. Seğmenoğlu M. S.: Streptomycin residue survey in honey. AVKAE Derg. 2013 3, 15-17.

30. Seidel M., Niessner R.: Chemiluminescence microarrays in analytical chemistry: A critical review. Anal. Bioanal. Chem. 2014, 406, 5589-5612.

31. Sunay A. E.: Problem of antibiotic residues in honey. U. Ar1 Drg. 2006, 4, $143-148$

32. Tamba-Berehoiu R., Visovan D. M., Popa C. N.: Study on the presence of antibiotic residues in honey intended for public consumption. Scientific Papers Series Management. Econ. Engin. Agric. Rural Develop. 2013, 13, 2285-3952.

33. Toldra F., Reig M.: Methods for rapid detection of chemical and veterinary drug residues in animal foods. Trends Food Sci. Technol. 2006, 17, 482-489.

34. Wutz K., Niessner R., Seidel M.: Simultaneous determination of four differen antibiotic residues in honey by chemiluminescence multianalyte chip immunoassays. Microchimica Acta 2011, 173, 1-9.

35. Zai I. U. M., Rehman K., Hussain A., Shafqatullah.: Detection and quantification of antibiotics residues in honey samples by chromatographic techniques. Middle East J. Sci. Res. 2013, 14, 683-687.

Corresponding author: Assist. Prof. Dr. Aksem Aksoy, Kafkas University, Faculty of Engineering Architecture, Department of Food Engineering, TR36100, Kars, Turkey; e-mail: aksemaksoy@hotmail.com 